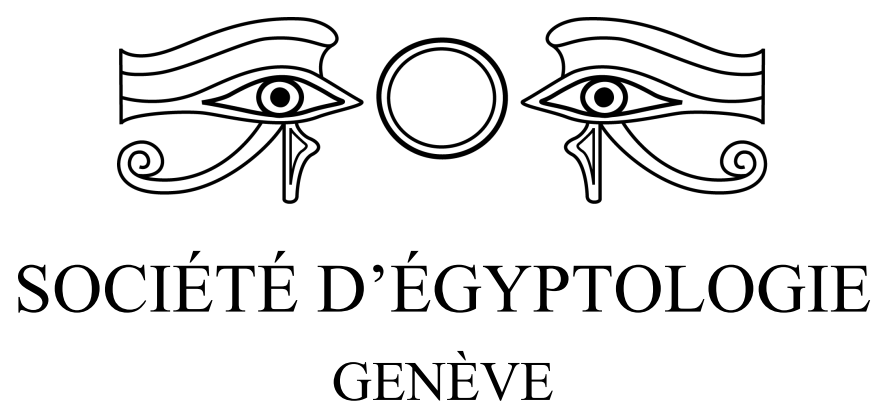

BULLETIN N 32

2021

\title{
Jean-Jacques Rifaud à Genève
}

\section{Jean-Luc CHAPPAZ}

Musée d'art et d'histoire de Genève

jean-luc.chappaz@bluewin.ch

\section{Résumé}

Jean-Jacques Rifaud (1786-1852) est connu par ses découvertes pionnières et par quelques publications assez maladroites. Il laisse également d'importantes archives, dont quelques milliers de feuillets nouveaux ont été récemment portés à l'inventaire de la Bibliothèque de Genève. Outre l'Égypte, ces documents concernent aussi Venise, la Belgique, la Hollande et la Suisse. Après une présentation rapide du contenu des archives égyptiennes, cet article s'attarde plus particulièrement sur ses descriptions de Genève, mettant en exergue la personnalité bipolaire de l'auteur, ses prétentions, ainsi que le manque d'originalité de ses observations.

Mots-clés : Jean-Jacques Rifaud; archives ; Genève; histoire de l'égyptologie; James Fazy ; Guillaume-Henri Dufour
Rue J.-F. Bautte 9

CH-1201 Genève (Suisse)

\begin{abstract}
Jean-Jacques Rifaud (1786-1852) is known for its pionnering discoveries and for a few publications fairly clumsy. He also left some important archives, of which a few thousand new pages have recently been added to the inventory of the Bibliothèque de Genève. In addition to Egypt, these documents also concern Venice, Belgium, Holland and Switzerland. After a brief presentation of the Egyptian archives, this article focuses on his descriptions of Geneva, highlighting the author's bipolar personality, his pretensions, and the lack of originality of his observations.
\end{abstract}

Keywords: Jean-Jacques Rifaud; archives; Geneva; history of egyptology; James Fazy; Guillaume-Henri Dufour

\section{Comment citer/How to cite}

Jean-Luc CHAPPAZ, « Jean-Jacques Rifaud à Genève », BSÉG 32 (2021), pp. 5-31. 


\title{
Jean-Jacques Rifaud à Genève
}

\author{
Jean-Luc CHAPPAZ
}

\begin{abstract}
Jean-Jacques Rifaud (1786-1852) est connu par ses découvertes pionnières et par quelques publications assez maladroites. Il laisse également d'importantes archives, dont quelques milliers de feuillets nouveaux ont été récemment portés à l'inventaire de la Bibliothèque de Genève. Outre l'Égypte, ces documents concernent aussi Venise, la Belgique, la Hollande et la Suisse. Après une présentation rapide du contenu des archives égyptiennes, cet article s'attarde plus particulièrement sur ses descriptions de Genève, mettant en exergue la personnalité bipolaire de l'auteur, ses prétentions, ainsi que le manque d'originalité de ses observations.
\end{abstract}

Parmi les pionniers qui explorèrent le sous-sol archéologique de l'Égypte au début du XIX ${ }^{\mathrm{e}}$ siècle, Jean-Jacques Rifaud est sans doute l'un des plus mal connus et l'un des plus controversés. Contrairement à ses « rivaux » (Giovanni Battista Belzoni [1778-1823] et son épouse Sarah [1783-1870], ou Johann Ludwig Burckhardt [1884-1817]), qui croisent son chemin en Thébaïde, Rifaud n'a pas publié le récit de sa vie en Orient, ni de son vivant, ni à titre posthume. Il n'a pas le talent - pas plus que la formation - d'un Pascal Coste (1787-1879) ni d'un Frédéric Caillaud (1787-1869), personnalités qui figurent en bonne place dans les listes des voyageurs rencontrés en Égypte qu'il dressa probablement à son retour en Europe $^{1}$. Il a encore moins l'aura diplomatique ou politique des consuls

\footnotetext{
* Il m'est agréable de remercier Mmes Barbara Roth-Lochner et Paule Hochuli Dubuis, conservatrices du Département des manuscrits de la Bibliothèque de Genève, pour l'accès à ces documents, M. Roger Rosset (Archives d'État de Genève), ainsi que Mme Melissa Mihail et M. Pierre-Alain Baudat (Bibliothèque de Genève) pour son accueil cordial en salle de consultation.

Dans les citations, les propos de Rifaud sont reproduits au mieux de leur déchiffrement, en renonçant à indiquer par " sic » les graphies erronées ou les fautes de syntaxe. Dans les longues citations, le signe «|» indique la fin d'une ligne, «\|» le passage intentionnel à un nouveau paragraphe ; le sigle « $<$ ? > » signale une incertitude dans l'établissement du texte.

${ }^{1}$ Le Département des manuscrits de la Bibliothèque de Genève (ci-après : BGE) possède deux listes de la main de Rifaud recensant les personnalités croisées durant son séjour en Égypte (Ms. fr. 7863, env. 29), qui totalisent 175 noms (voir l'analyse de M.-C. BRUWIER, « Les rencontres de JeanJacques Rifaud en Égypte » dans M.-C. Bruwier, W. Claes \& A. QuertinMont, « La Description de l'Égypte » de Jean-Jacques Rifaud (1813-1826) (Connaissance de l'Égypte Ancienne 16), Bruxelles, éditions Safran, 2014, pp. 8-29 (ci-après : M.-C. BruWIER, W. ClaES \& A. QUERTINMONT,
} 
Bernardino Drovetti (1776-1852), Henry Salt (1780-1827) ou Giovanni Anastasi (1780-1860), qu'il nous dit avoir également côtoyés, et dont les noms sont associés à la création des grandes collections européennes, mais dont l'intérêt pour l'archéologie cède vite le pas aux considérations commerciales que représentait pour eux la vente des collections.

Décédé sans héritier à Genève le 9 septembre 1852, Rifaud laisse des centaines de dessins et des milliers de feuillets manuscrits, vendus à l'encan en mars 1853 , et aujourd'hui conservés après diverses péripéties au Département des manuscrits de la Bibliothèque de Genève. De son vivant, il avait déjà exploité une partie de cette documentation. Ainsi paraissait sous son nom un Tableau de l'Égypte, de la Nubie et des lieux circonvoisins ou itinéraire à l'usage des voyageurs qui visitent ces contrées (Paris 1830), premier "guide touristique » consacré à ces régions, dont le contrat d'édition précisait toutefois que la mise en forme du texte était confiée à un rédacteur extérieur ${ }^{2}$. Un petit fascicule publié par la Société de Géographie relate les fouilles que Rifaud avait entreprises dans le Fayoum à Kom Médinet el-Fares ${ }^{3}$, texte assez délicat à suivre aujourd'hui, en raison d'une certaine confusion de la description architecturale, par ailleurs noyée sous une abondance de précisions dimensionnelles ${ }^{4}$ (épaisseur des murs, largeur et longueur des pièces, hauteur de certains éléments, proportion des briques, etc.). Ces publications lui valurent quelque notoriété. Son œuvre majeur - celui auquel il ne cessa de croire jusqu'à ses derniers jours - reste cependant son Voyage en Égypte, en Nubie et lieux circonvoisins, trois volumes de planches accompagnés de volumes de texte, édition entreprise par deux fois et restée inachevée. Seules 222 planches sur les 300 prévues parurent en plusieurs livraisons, assez espacées dans le temps et mal diffusées ${ }^{5}$, et les commentaires restèrent lettre morte, au point qu'on s'est longtemps demandé s'ils n'avaient jamais été rédigés.

Description Rifaud). Parmi d'autres rencontres : Lord Belmore (1774-1841) ou le comte Auguste de Forbin (1777-1841), dont les « souvenirs » rapportés de leurs voyages contribuèrent à l'extension des collections du British Museum et du Louvre ; Giuseppe Passalacqua (1797-1865) et le général prussien d'origine genevoise Heinrich von Minutoli (1772-1846), «créateurs » du fonds égyptien du Musée de Berlin. Biographies succinctes de ces personnages dans M. L. BIERBRIER, Who Was Who in Egyptology, Londres (4 édition) 2012, passim.

${ }^{2}$ BGE, document (copie ou brouillon annoté) inséré dans Ms. suppl. 111, f 72.

${ }^{3}$ Description des fouilles et des découvertes faites par M. Rifaud dans la partie est de la butte Koum-Medinet-el-Farès lue à la Société de Géographie, le vendredi 19 juin 1829 (le manuscrit original du texte publié est conservé à la BGE sous la cote Ms. fr. 7863, env. 20).

${ }^{4}$ Rifaud n'utilise quasi jamais le système métrique : il parle en lieues, pieds, pouces et lignes, mesures qui variaient selon les régions, et se dispense de mentionner à quel système il fait référence.

${ }^{5}$ W. CLAES, «Les lithographies de Jean-Jacques Rifaud. Un voyage à travers l'Europe » dans M.-C. Bruwier, W. Claes \& A. Quertinmont, Description Rifaud, pp. 35-44. 
Les textes du Tableau sont souvent vagues, voire confus, particulièrement lorsqu'ils abordent l'Antiquité ou des propositions d'itinéraires. Est-ce le fait de Rifaud ou de son « rédacteur »? Quant aux planches du Voyage, elles sont d'une maladresse et d'une imprécision affligeantes, à l'exception de quelques plans de temples cotés et levés à une échelle constante. Mais que dire des copies hiéroglyphiques, des proportions des bas-reliefs ou des statues, que seules la bonne volonté de l'observateur ou l'histoire des collections permettent d'identifier partiellement? C'est ainsi, avec ce préavis fort négatif, que quelques chercheurs redécouvrirent Rifaud à la fin $\mathrm{du} \mathrm{XX}^{\mathrm{e}}$ siècle, ce qui les amena à prendre en considération les archives inédites alors disponibles. Sans surprise, les centaines de dessins confirmaient la médiocrité de leur auteur pour cet art, amplifiée sans doute par la difficulté à s'approvisionner en papier ${ }^{6}$. Plus rédhibitoires encore, trois volumes manuscrits faisaient partie du lot conservé, connus sous le titre de 《 mémoires $»^{7}$ (couvrant essentiellement les principaux épisodes de ses errances jusqu'en 1832). Partiellement redondants, et même s'ils permettaient de retracer une partie de la vie de l'explorateur, ces textes révélaient d'autres facettes peu flatteuses du personnage : si son écriture, quelque peu archaïque ${ }^{8}$, est globalement parfaitement lisible, Rifaud était un quasi illettré ! Son orthographe est non seulement inconstante, le plus souvent phonétique, mais le découpage même des mots correspond plus souvent à la transcription de sons qu'à celle du sens ${ }^{9}$. Ponctuation et syntaxe vont de pairs, les répétitions foisonnent. Curieusement néanmoins, le vocabulaire de Rifaud n'est pas pauvre et on trouve sous sa plume des termes relativement savants ou spécialisés (même si mal orthographiés), preuves sans doute d'une réelle curiosité et d'une certaine mémoire, due à la fréquentation de cercles cultivés dont il aura retenu les propos. Mais, beaucoup plus inquiétant, l'auteur rédige ses mémoires sur un ton obsessionnel, si ce n'est maladif. Il se plaint de son impécuniosité, qu'il attribue aux ladreries du consul de France, Bernardino Drovetti, pour le compte duquel (ou en association avec lequel, selon Rifaud) il a travaillé en Égypte. Il détaille les profits que le consul a réalisés en vendant ses collections ${ }^{10}$, l'accusant de l'avoir payé en monnaie de

\footnotetext{
${ }^{6}$ Difficulté persistante tout au long de sa vie, car l'ensemble de ses archives a pour support des papiers de toute qualité, de tout format et de toute couleur!

${ }^{7}$ BGE, Ms. suppl. 111 à 113.

${ }^{8}$ Par exemple, il utilise encore le «s long » $(>\mathrm{f})$.

${ }^{9}$ Par exemple, BGE, Ms. suppl. 113, fo 43 : «M. | Drovetti eilliant jetes un coup deuil sur mes capasite | et voÿant lamour que je maitais au travail et atout | se que jantrepenet, il mangajet bocoup a me randre | au Caire pour etre an ploÿes au pre de Mehemet a | lÿbacha an efet sete au casion me fut favorable M. | drovetti netet plus consul ».

${ }^{10}$ Pour les œuvres les plus prestigieuses (et les plus pesantes), voir S. CinCOTTI, «'Les fouilles dans le Musée' : la collection égyptienne de Turin et le Fonds Rifaud », Cahiers de Karnak 14
} 
singe alors qu'il lui constituait, sur le terrain, la matière de sa fortune. D'autres rancunes, notamment vis-à-vis de Belzoni, rappellent la rivalité de ces chasseurs de trésors. En fait d'autobiographie, les "mémoires» de Rifaud ne sont qu'un monotone et répétitif réquisitoire, développé sur plus de sept cents pages et prenant le plus souvent la forme de lettres (réelles ou fictives), par lesquelles il espérait peut-être exorciser le « monstre » qu'était à ses yeux le consul Drovetti.

La date de rédaction de ces trois mémoires est inconnue ${ }^{11}$, comme sont approximatives certaines étapes de la vie de Rifaud à partir de son retour en Europe.

Nonobstant, quelques chercheurs reconnurent l'importance de Rifaud pour l'histoire de la redécouverte de l'Égypte. Jean-Jacques Fiechter intégrait ce personnage dans sa Moisson des dieux, et lui redonnait l'importance qui fut la sienne dans la formation des grandes collections européennes ${ }^{12}$. Marie-Cécile Bruwier, profitant d'avoir entre les mains une des éditions les plus complètes du Voyage, organisait quelques années plus tard une exposition à Nivelles ${ }^{13}$, associant à son projet de nombreux chercheurs, qui mirent en avant l'originalité de quelques observations ou détails qui ne sont rapportés que par l'œuvre de Rifaud. En 2012, Michel Azim lui consacre un long chapitre dans sa Topographie de Karnak ${ }^{14}$. Deux ans plus tard, Marie-Cécile Bruwier reprenait le dossier et, associée à deux collègues, présentait une réédition de toutes les planches connues du Voyage, avec un commentaire contemporain érudit ${ }^{15}$. Auparavant, dans une succession d'articles, Jean Yoyotte ${ }^{16}$, sans doute le plus sévère dans son appréciation de l'œuvre de Rifaud, avait utilisé certains de ses dessins pour retrouver l'origine de monuments, et insistait avec raison sur l'originalité de ses «reportages » relatifs à l'Égypte du $\mathrm{XIX}^{\mathrm{e}}$ siècle et sur l'intérêt de cette

(2013), pp. 279-285 ; J.-J. FIECHTER, La Moisson des dieux. La constitution des grandes collections égyptiennes, Paris (Julliard) 1994, pp. 98-103 et 233-236.

${ }^{11}$ On y lit toutefois la date de 1842 , notamment BGE, Ms. suppl. 111, $\mathrm{f}^{\circ}$ 64-65.

12 J.-J. FieChter, La Moisson des dieux, Paris 1994, passim, voir p. 287 (index).

${ }^{13}$ L'Égypte au regard de J.-J. Rifaud, Lithographies conservées dans les collections de la Société royale d'Archéologie, d'Histoire et de Folklore de Nivelles et du Brabant Wallon, Nivelles 1998.

${ }^{14}$ M. AzIM, Karnak et sa topographie. Les relevés anciens du temple d'Amon-Rê de 1589 aux années 1820, Paris 2012, vol. 2, pp. 293-324, avec une riche bibliographie.

15 M.-C. Bruwier, W. Claes \& A. Quertinmont, Description Rifaud, avec une bibliographie exhaustive, pp. 285-288. Ajouter depuis lors : A. Delattre \& N. VANTHIEGHEM, « Une inscription disparue du Dayr al-Fahūūī », BIFAO 114 (2014), pp. 149-154.

16 « À la recherche d'un explorateur marseillais disparu», dans D. JACOBI et alii (éds), Pascal Coste. Toutes les Égypte (exposition à la Bibliothèque municipale, 26 mars - 20 juillet 1998), Marseille 1998, pp. 221-234 ; "Le grand Kôm el-Ahmar de Menûfîyah et deux naos du pharaon Amasis », BSFE 151 (juin 2001), pp. 54-81; «Jean-Jacques Rifaud (1786-1852), un singulier voyageur qui vint mourir à Genève ", dans J.-L. CHAPPAZ et Cl. RiTsChARD (éds), Voyages en Égypte de l'Antiquité au début du XX' siècle (exposition au Musée d'art et d'histoire, 16 avril - 31 août 2003), Genève 2003, pp. 87-97. 
documentation à une meilleure connaissance du pays à l'époque de Méhémet-Ali. Il doute toutefois de l'honnêteté et de la sincérité de l'auteur qui chercherait à dissimuler (ou à protéger pour son propre intérêt) certaines informations archéologiques, le qualifiant notamment d' $"$ homme de terrain, inculte mais actif, pauvre mais intrépide », de «misérable [qui] raconte confusément [ses] exploits (...) et [ses] souffrances (...) tout en remâchant indéfiniment ses rancunes », aux « dessins (...) laids et maladroits (...) fantaisistes (...) pénibles à voir », ou encore de $«$ fieffé menteur ${ }^{17}$.

À ce stade de l'enquête, la biographie de Rifaud se réduit à quelques rares éléments. Il naquit à Marseille le 29 novembre 1786, d'une famille d'artisans aisés mais bientôt ruinée par la Révolution. Il reçoit une éducation minimale de la part d'un curé, se lance dans un apprentissage de sculpteur sur bois et entreprend son «tour de France ». Il est rattrapé par la conscription, fait prisonnier (ou déserte) en Espagne, se lie à des exilés royalistes et peut-être maçonniques, avant de premières pérégrinations à travers la Méditerranée. En juillet 1813, il débarque à Alexandrie et se place sous la protection du consul de France Bernardino Drovetti qui le recommande à Méhémet-Ali. En 1816-1817, il remonte le Nil au-delà de la Deuxième Cataracte et il est l'un des premiers explorateurs occidentaux, si ce n'est le premier, à décrire et dessiner les temples de Basse Nubie ${ }^{18}$ (fig. 1). Au retour, il s'installe à Thèbes où le consul Drovetti le charge de conduire les fouilles fructueuses qui amèneront à la constitution de sa première collection (vendue à Turin). Parallèlement, Rifaud part à la découverte du pays, y observant les curiosités en matière de sciences naturelles, d'architecture, de mœurs, etc., en anthropologue naïf qu'il était.

Son retour en France (1827) lui vaut quelques succès, dont la légion d'honneur, et diverses associations académiques ou savantes lui prêtent une oreille attentive, sans toutefois s'engager davantage à la publication de ses travaux. La première tentative d'édition de son Voyage tourne court, car les Trois Glorieuses ont fait fuir ses souscripteurs. Rifaud (re)commence alors une vie d'errance à travers l'Europe pour promouvoir son œuvre, recevra un bon accueil à la cour de Russie

\footnotetext{
${ }^{17}$ Citations extraites de «Le grand Kôm el-Ahmar de Menûfîyah et deux naos du pharaon Amasis », BSFÉ 151 (juin 2001), pp. 54-81.

${ }^{18}$ Il précède en effet Frédéric Caillaud et surtout l'architecte François-Chrétien GAU qui aura la primeur de publier ces temples : Antiquités de la Nubie, ou Monumens inédits des bords du Nil, situés entre la première et la deuxième cataracte (sic), Stuttgart-Paris 1822.

Rifaud réalisa toutefois une douzaine de maquettes de ces temples, parvenues à Turin en 1823 avec la première collection Drovetti. Sur cette attribution : S. EINAUDI, « Les maquettes des temples nubiens du Musée égyptien de Turin. Une mise au point », dans M.-C. BRUWIER, W. ClaES \& A. Quertinmont, Description Rifaud, pp. 30-34.
} 


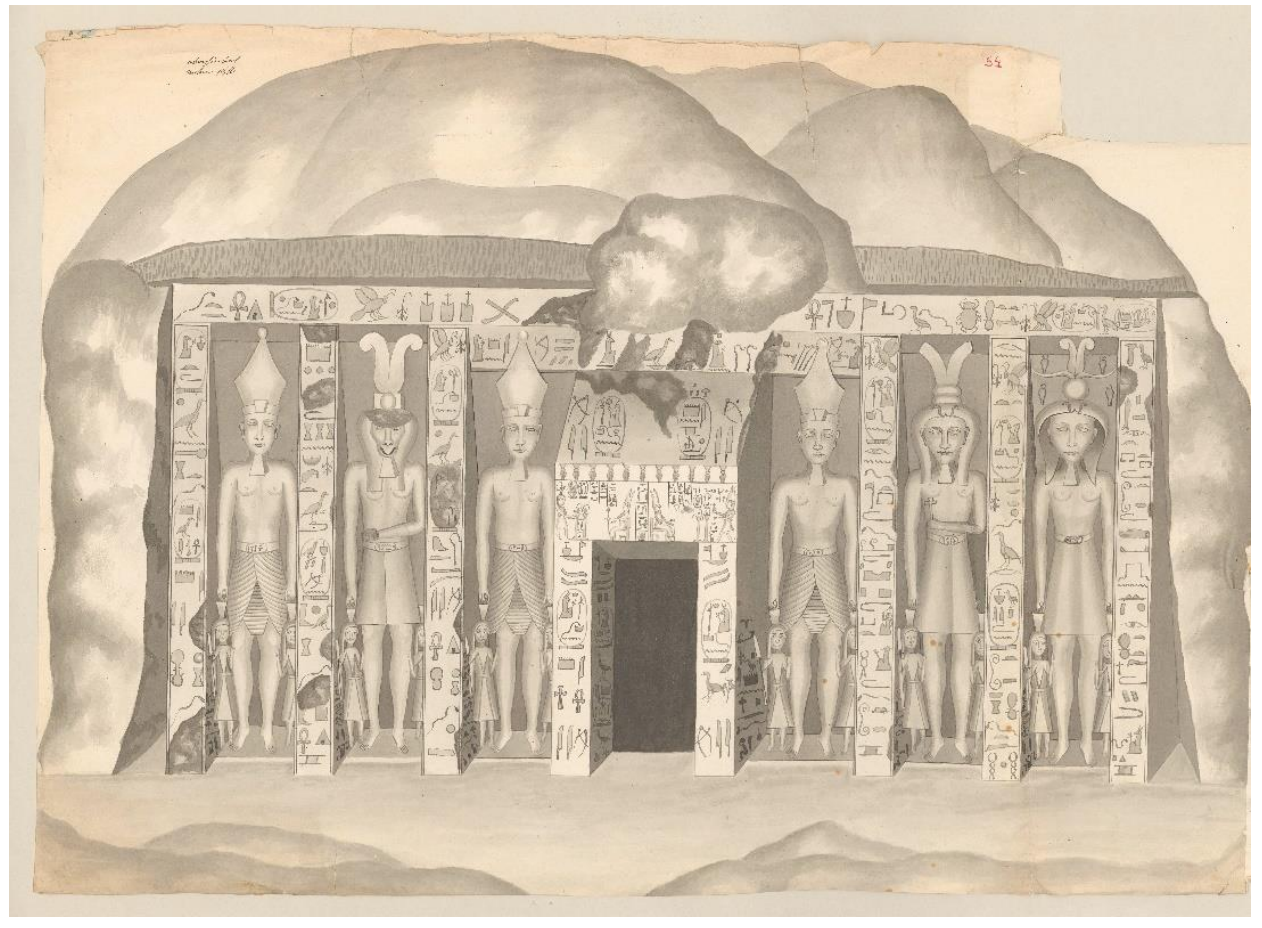

Fig. 1 : Dessin de la façade du petit temple d'Abou-Simbel par Rifaud en 1816-1817 (C) Bibliothèque de Genève, Ms. fr. 1602/1, f $54 \mathrm{r}^{\circ}$, détail)

(1837), ce qui lui permettra de lancer la « seconde » édition de son projet. Mais, sans cesse désargenté, il n'en vint jamais à bout et dut poursuivre ses incessants déplacements à la recherche de soutiens qu'il ne trouva point.

Comme l'avait annoncé discrètement Jean Yoyotte en 2001, ce portrait de JeanJacques Rifaud peut être à présent nuancé ${ }^{19}$. Des milliers de « nouveaux » feuillets ont été portés à l'inventaire du Département des manuscrits de la Bibliothèque de Genève, tâche menée à bien par Mme Aurélie Barjon-Cendre en $2005^{20}$. À défaut de lever tous les doutes et de permettre la narration d'une biographie linéaire, ces documents, joints aux «mémoires » déjà connus, permettent de préciser l'itiné-

\footnotetext{
n. 2 .

${ }^{20}$ A. BARJON-CENDRE, « Redécouverte de Jean-Jacques Rifaud au Département des manuscrits », dans : La Bibliothèque de Genève en 2005, 2006 et 2007, Genève, Ville de Genève, Département de la Culture, 2009, pp. 9-14. L'ensemble du fonds est catalogué sous : http://w3public.ville-ge.ch/bge/ odyssee.nsf/Attachments/rifaud jeanjacquesframeset.htm/\$file/rifaud jeanjacques.htm (consulté le 23.10.2020).
}

19 « Jean-Jacques Rifaud (1786-1852), un singulier voyageur qui vint mourir à Genève », p. 97, 
raire de Rifaud et de relativiser l'importance de cette documentation. Il est vrai que le jugement catégorique de Jean Yoyotte, que confirme un rapide examen des documents relatifs à l'Antiquité, oblige à nuancer l'intérêt de cette (re)découverte et invite à émettre des doutes quant à l'apport scientifique, stricto sensu, de ces abondantes liasses pour l'étude de l'archéologie égyptienne.

\section{Nouveaux documents}

L'essentiel de cette archive concerne l'Égypte (env. 3700 feuillets), où Rifaud vécut de 1813 à 1826. Le principal étonnement est que l'écriture (et donc l'orthographe et la syntaxe, clairement intelligibles) n'est pas celle de Rifaud : il aura bénéficié de l'aide de plusieurs rédacteurs extérieurs ${ }^{21}$. Une certaine redondance peut également être observée, quelques documents ayant servi de « souches » à la production d'autres textes. On y trouve deux « itinéraires », sorte de journal de bord (plus qu'intime) sur la vie de Rifaud à Thèbes en 1821-1823, puis dans le Fayoum et le Delta (1824-1825), avec quelques indications de son activité archéologique, mais les considérations sur ses déplacements, la population, l'actualité telle qu'elle lui parvient, la flore, la faune, les mœurs, les ressources agricoles (et industrielles) dominent largement. Un autre ensemble rassemble des relevés météorologiques (deux copies d'une même source). Éparses, quatre dossiers regroupent des «notes » sur respectivement la Nubie, l'Égypte, la province du Fayoum et le Delta ${ }^{22}$. Figurent également en bonne place une partie des notices préparées comme commentaires aux planches du Voyage, et restées à ce jour inédites ${ }^{23}$ (par ordre croissant de notices : poissons, antiquités, costumes, autres animaux, botanique).

Les propos consignés sont surtout des témoignages, parfois un peu naïfs, de la réalité observée par Rifaud. Il n'approfondit guère, constate, et souvent se révolte contre la rapacité du pouvoir de Méhémet-Ali et de ses sbires, la corruption, la violence qui règne alors dans le pays, l'oppression constante à l'égard des fellahs. Il ridiculise les superstitions des populations. Il n'hésite guère à se mettre en scène, apportant réconfort et secours aux malheureux, de façon souvent romanesque ${ }^{24}$. Il

21 Plusieurs « mains » interviennent dans ces textes, dont quatre « principales ». On en observe d'autres, mais généralement en marge, pour des feuillets additionnels ou dans la copie de rapports d'institutions académiques.

${ }^{22}$ Les titres des dossiers sont de l'écriture de Rifaud. Toutefois, le matériel regroupé dans chacun de ces dossiers est épars et bien souvent sans rapport avec cet apparent classement.

${ }^{23}$ Le rapport entre planches et textes relève de la quadrature du cercle ! L'œuvre étant inachevée, il faut donc admettre des planches publiées restées sans commentaires, et des commentaires préparés pour des planches non encore réalisées!

${ }^{24}$ Pour une analyse plus complète du contenu de ces archives et de l'attitude du narrateur, voir J.L. ChAppaZ, « Autobiographies rocambolesques. À propos de quelques manuscrits inédits de Jean- 
est possible qu'un historien de l'Égypte du XIX ${ }^{\mathrm{e}}$ siècle puisse trouver, dans cette abondante documentation, des sources qui, après recoupements, lui permettront d'ébaucher une analyse de la situation sociale (et de la misère) des campagnes nilotiques à cette époque. Les remarques de l'auteur en matière de science naturelle paraissent moins pertinentes. Quant à l'Antiquité, elle n'occupe qu'une infime part, congrue, de cet ensemble: ses commentaires aux planches sont minimalistes (pouvait-il en être autrement alors que la redécouverte de la civilisation pharaonique en était à ses balbutiements ?), et il ne dit quasi rien de ses propres fouilles. De menues remarques peuvent toutefois être glanées en marge du dossier sur le Fayoum ${ }^{25}$, la Nubie ${ }^{26}$ ou le Delta, où se trouvent quelques pages consacrées à :

- Tanis $(\ll \text { San } »)^{27}$

- Tell Moqdam / Léontopolis (« Moukedam »/ « coum mougédam ») 28

- Tell Atrib / Athribis (« Com-Talle-Trip ») $)^{29}$

- Tell Basta / Bubastis (« Télébaste ») $)^{30}$

- Menûfiyah / Kom el-Ahmar (« Coum Larmar ») $)^{31}$

Des références à l'ouvrage de Pococke ${ }^{32}$ publié en $1743-1745$, ou à la Description de l'Égypte (édition impériale) à propos du temple d'Esna ${ }^{33}$, comme

Jacques Rifaud », dans S. HAUPT, C. SPIESER \& M. VIEGNES (éds), "Ton rêve est une Égypte... » "Dein Traum ist ein Ägypten... " Ägyptomanie in Kunst und Literatur L'égyptomanie dans la littérature et les arts. Colloque Université de Fribourg, 5, 6 et 7 mars 2020, Würzburg 2021, sous presse.

${ }^{25}$ BGE, Ms. fr. 7865, env. 1 : remarques sur les pyramides d'el-Lahoun («Laoun ») et d'Hawara («Lavouhara »); $\mathrm{f}^{\circ} 24-27$ : description du temple de Qasr-Qaroun (« Caroun »); $\mathrm{f}^{\circ} 65$ : bref survol de la nécropole de Saqqarah («Saccarah »), puis de Tell Athrib / Athribis («tale trib », fo 90) et, au passage, de rapides suggestions sur des sites du Delta où des fouilles prometteuses pourraient être conduites ( $\mathrm{f}^{\circ}$ 61, 91, 96, 99). Voir aussi pour ses fouilles au Fayoum, BGE, Ms. fr. 7868, env. 6.

${ }^{26}$ BGE, Ms. fr. 7866 : outre la mention de plusieurs monuments, quelques pages sont consacrées (fo 347-350) aux objets funéraires qu'on trouve à Thèbes.

${ }^{27}$ BGE, Ms. fr. 7869 , env. 20.

${ }^{28}$ BGE, Ms. fr. 7869, env. 21.

${ }^{29}$ BGE, Ms. fr. 7872, env. 4.

${ }^{30}$ BGE, Ms. fr. 7869, env. 14.

${ }^{31}$ BGE, Ms. fr. 7872, env. 4, $\mathrm{f}^{\circ}$ 59-61. Non sans intérêt, Rifaud fait noter ( $\left.\mathrm{f}^{\circ} 60\right)$ qu' « il s'y découvrit deux monolytes en granit rose dont le sommet était en forme pyramidale; un de ces monolyte était d'une grande dimension, et orné de toutes parts de cartouches et de sujets hiéroglyphiques, ce monument fut tiré de ce lieu en 1826 pour le compte de M. Danastase Consul de Suède qui se trouve aujourd'hui au musée Royal de Suède ». Cette remarque vient confirmer définitivement l'intuition de J. YOYOTTE quant à la provenance du naos au nom d'Amasis conservé au Musée de Leyde («Le grand Kôm el-Ahmar de Menûfîyah et deux naos du pharaon Amasis », $B S F E$ 151, juin 2001, pp. 54-81).

32 BGE, Ms. fr. 7866, fo 253 (« Pockocke »).

${ }^{33}$ BGE, Ms. fr. 7872, env. 1, fo 7 : «Je ne donne que le détail des Chapiteaux, vu que le dessin de ce temple a été donné par l'ouvrage de la Commission d'Egypte ». 
la mention d'une correspondance entretenue avec une personnalité cairote en $1842^{34}$, tendent à prouver que Rifaud avait accès à une information minimale et qu'il travaillait à la rédaction de son ouvrage. Plus curieux, le manuscrit de ses commentaires aux planches d'antiquités se termine par un feuillet recto-verso ${ }^{35}$ dans lequel l'auteur appelle à la conservation des sites archéologiques, dénonce les vandalismes qui s'y opèrent et énumère une certain nombre de destructions dont il aurait été le témoin: "Tant que ce pays se trouvera sous un tel gouvernement il y a à craindre qu'une grande | partie des monumens qui existent encore debout ne deviennent au rang de ceux | qui ne restent plus que leur emplacement, un peu le nil et le barbarisence de / ce peuple ont détruit depuis 1815 $<$ ?> une quantité d'édifices qui auraient certainement | encore existé un grand nombre de siècle car depuis gash à disparu les restes de | Cheik abadÿ, ceux d'achmounein celui de contrelatopolis, les deux petits temples | de el Kab-elethÿa dont les matériaux de ces trois monumens ont servi pour | consolider le quai d'esné, les deux temples de l'ile éléphantine ont été entièrement | détruits pour servir à la Construction d'un hôpital militaire, enfin il y a à $\mid$ des projets pour établir des salpètrières au soleil ». Cet extrait suscite deux remarques. Tout d'abord, le temple de Contralato, dont une élévation, un écorché et le plan sont publiés dans les planches imprimées du Voyage ${ }^{36}$, n'a été détruit qu'en novembre $1828^{37}$, soit après le départ d'Égypte de l'auteur. Mais surtout, l'ordre dans lequel ces monuments disparus sont énumérés se retrouve dans un texte autrement plus célèbre: la Note remise au Vice-Roi pour la conservation des monuments de l'Égypte, que Jean-François Champollion prépara à l'attention de Méhémet-Ali en novembre 1829, sans doute diffusée peu après et reproduite dans les Lettres écrites d'Égypte et de Nubie rassemblées par le frère du déchiffreur en $1833^{38}$. Si un tel extrait (pour ne pas dire plagiat) prouve le zèle que Rifaud mettait à poursuivre son projet, il oblige son lecteur à s'interroger sur l'originalité, voire l'authenticité et la sincérité de son œuvre.

\footnotetext{
${ }^{34}$ BGE, Ms. fr. 7867, env. 2, fo 26 : « Le canal du Barh ÿousouf reouvret (sic) en 1842 | dans la partie de la haute Egÿpte (...) Mon correspondant de Caire m'écrivit en 1842 (...) ».

${ }^{35}$ BGE, Ms. fr. 7872, env. 3, $\mathrm{f}^{\circ}$ 57, dont le titre « Notes sur les dégradements des monuments de légypte » est cependant biffé.

${ }^{36}$ M.-C. Bruwier, W. Claes \& A. Quertinmont, Description Rifaud, pp. 114-166 (notices de Claude Obsomer et Florence Doyen).

${ }^{37}$ Lettre de Jean-François Champollion du 8 décembre 1828. Réédition d'après les collations de Hermine HARTLEBEN (1909) chez Christian Bourgeois éditeur, Paris 1986, p. 168.

${ }^{38}$ IBID., pp. 443-448. Champollion cite successivement : « tous les monuments de Chéik-Abadé », «le temple d'Aschmounéïn », le "temple de Kaou-el-Kebir », un "temple au nord de la ville d'Esné », un « temple vis-à-vis Esné, sur la rive droite du fleuve », « trois temples à El-Kab ou ElEitz », « deux temples dans l'île, vis-à-vis la ville d'Osouan, Géziret-Osouan » (p. 445).
} 


\section{Retour en Europe}

Suivant les itinéraires empruntés par Rifaud à son retour en Europe (c'est lui qui reprend la plume, l'orthographe et la syntaxe), se développent chronologiquement près de deux milliers de feuillets sur Venise, où il vécut en 1841-1842, la Hollande (1835 et 1845) ; la Belgique (1842 et plus tard ?) et enfin une traversée de la Suisse (448 feuillets) qui s'achève à Genève en été 1852 (90 feuillets).

Grâce à ces documents, nous sommes renseignés par Rifaud lui-même sur de nombreuses étapes de sa vie, mais d'autres larges parts restent dans l'ombre. De rares informations nous apprennent en effet que Rifaud se rendit en Angleterre vers 1832, à la recherche de souscripteurs pour la publication de son Voyage, puis en Russie (puisque, après Louis-Philippe, le tsar Nicolas $I^{\text {er }}$ accepta de patronner l'ouvrage), en Bavière, où les planches de la seconde édition furent imprimées (dès 1837). Il est également délicat de suivre ses pérégrinations entre Venise (qu'il quitte en 1842) et les Pays-Bas (qu'il rejoint pour un second séjour en 1845).

Dans cette errance européenne, les remarques de Rifaud sont constituées d'une suite de notices relativement brèves, à l'image de ses observations sur l'Égypte. Sous divers intitulés (qui ne correspondent pas toujours au texte développé), il évoque la topographie, les monuments, la météorologie, la qualité des eaux, l'industrie et l'agriculture, les gouvernements, les populations, l'actualité, la flore et la faune, l'état sanitaire, les constructions et l'urbanisme, les promenades, la vie culturelle, la justice et les injustices (et il s'érige très souvent en « champion » des victimes) en criant haut et fort son indignation. À cet égard, Rifaud est un individu sensible, prêt à s'émouvoir et à se faire le porte-parole de causes qui lui importent manifestement, mais qu'il ne dénonce - faute de mieux, mais non sans conviction - que face à lui-même, sans en chercher les origines et sans développer une argumentation si ce n'est construite, du moins étayée.

Le propos qui sera développé ci-dessous se limite à une analyse des notices que Rifaud consacre à Genève, durant son bref séjour de l'été 1852. Partant du principe que les témoignages qu'il donne de la réalité de l'Égypte sous le règne de Méhémet-Ali ou que ses notes anthropologiques sont les aspects les plus originaux (voire dignes de la plus grande attention) développés par Rifaud, cette enquête répond à un seul objectif : essayer de définir l'intérêt de ce lot d'archives à partir d'un exemple bien documenté par ailleurs, non soumis à notre ignorance des caprices des politiques coloniales que les grandes puissances exerçaient sur l'Égypte à l'époque où Rifaud y vivait. Elle a l'avantage de ne reposer que sur une 
petite centaine de feuillets manuscrits - tous de la main de Rifaud - qui permettent aisément de repérer redites et contradictions. Outre leur intérêt - somme toute très relatif - pour l'histoire genevoise, ses conclusions devraient permettre, par extrapolation, de relativiser l'apport et la qualité des archives Rifaud sur l'Égypte, et accessoirement sur Venise, la Hollande, la Belgique ou la Suisse.

Des objections peuvent facilement être dressées contre cette démarche : lorsque Rifaud aborde le rivage alexandrin, il est un jeune homme de vingt-six ans ; il quitte l'Égypte à trente-neuf ans et meurt à Genève peu avant son soixante-sixième anniversaire. En près de trente ans, l'homme peut avoir changé, gagné en expérience, développé des rancunes, perdu en curiosité. Par ailleurs, l'histoire politique de l'Europe n'est pas celle de l'Égypte, la documentation n'est pas la même. L'Égypte du début du XIX ${ }^{\mathrm{e}}$ siècle peut être considérée comme une terra incognita, qualificatif qui ne peut guère être appliqué au canton et à la ville de Genève...

Même s'il nous faut admettre que les résultats de cette enquête restent sujets à nuances, la «méthode Rifaud» permet d'éclairer, dans ce cas particulier, les limites de ses témoignages, sa personnalité, et finalement la crédibilité de ses affirmations.

\section{L'aventure genevoise}

La documentation que Rifaud nous laisse de son séjour genevois consiste en un petit carnet de 40 pages, et en une cinquantaine de feuillets indépendants, parfois assemblés, de divers formats et de diverses qualités de papier. La foliotation est celle retenue par le Département des manuscrits et correspond probablement à l'ordre dans lequel ces différents papiers ont été retrouvés : ce classement n'est pas chronologique et on ignore ce qui a procédé à leur actuelle disposition. Les textes du carnet sont souvent (mais non systématiquement) repris sur des feuillets indépendants et plus développés, ils pourraient être la source première de l'auteur. Peu de dates sont conservées (juillet $1852>$ août 1852). On peut en déduire que Rifaud arriva à Genève entre la fin du mois de juin et le début de juillet, et qu'il y résida (rue Winkelried, 6) jusqu'à son décès. C'est pourquoi le présent article ne cherchera pas à suivre une quelconque chronologie, mais examinera les propos de Rifaud de façon plus regroupée, à défaut de thématique.

Si l'on peut aisément comprendre que Rifaud, qui comptait au nombre des premiers explorateurs de l'Égypte, ait accumulé moult notes sur ce pays, il est $a$ priori plus étonnant de le voir développer tant de propos sur Venise, la Hollande, la Belgique ou la Suisse. Manifestement, sa plume le démange, et peut-être se sent-il l'âme d'un homme de lettres ? Il est vrai que ses observations sur l'Égypte 
ont retenu l'attention de nombreuses institutions scientifiques - dont aucune n'a cependant pris en charge leur publication -, ce qui pouvait laisser croire à un Rifaud, ambitieux et dont la naïveté a sans doute été largement flattée, qu'il était un témoin privilégié de son époque. Plaide en ce sens une narration de Rifaud qui nous relate les entretiens qu'il eut quelques jours avant sa disparition avec James Fazy (1794-1878), président du gouvernement genevois (fig. 2 et 3 ) :

" geneve mr james fazÿ, president de | la république de généve

» le 16 le 20 et le 24 àout 1852 | conferé avec le prémier majistrat de | la republique de génévé. mangajait à passer | lhiver à geneve et man gatait $<$ ? $>$ a continuer $\mid$ mes recherches sur cette partie de la Suisse $\mid$ pour compléter sur ce canton tel que le | venait de faire pour tous les autres, vu que disait $\mid$ il la Suisse netait quinparfaitemen connue | malgré quon usse publies tant de souvenirs | et dimpressions de voÿag quen efait netait | que des itineraires qui se repetait comme | les compilations ordinaires qui rabachants | toujour la maime chause puisque ils se $\mid$ repetans sans autre chause,

» et qun ouvrage fait sur le pied et | le plan que javais entre pris mancait | et serait dun haut intéré, avec toutes les | recherches que je venais de faire sur | chaques localites avec cette manière aussi | excrupuleuse, quil était conue par eux | qui mavait jugés en me prometant de souscrire | et fair sous crire sur ma publication de legÿpte $\mid<$ verso :> nubie et lieux sirconcvoisins, et quen $\mid$ passant lhiver dans le canton de geneve il $\mid$ me metrait en $\mathrm{xxx}^{39}$ de seconder en mes recherches

» ce premier magistrat du canton de généve | fut tout ala foi tres porté pour moi et a $\mid$ me seconder, dans mes travaux sur cette partie $\mid$ de leurope, connaissent disetil quon seneté $<$ ? $>\mid$ jusqua lors pass ases aucupé, naÿant toujour | tréte que les memes sujets, en lessent le | principal de coté qui interesse si hautement $\mid$ a connaitre, ${ }^{40}, \mathrm{mr}$ fazÿ, men couragé $\mid$ hautemen de continuer mes observations | sur ce paÿs que tres souvent les indigenes | negligent ne prouvent les memes sansations $\mid$ ni les memes impressions que létragér voue | a ce genre de recherches sur chaques localites. | pour completer des donnes plus positives.

» il me fit part aussi du bel établissement $\mid$ scientifique $^{41}$ quon alle construir a génév pour $\mid$ relever les sciances et les lettres ala dignité $\mid$ qui méritents, et que le plan en estait grandiose | et ÿ attirer des paÿs etrangér des hommes |

\footnotetext{
${ }^{39}$ Mot de trois lettres, illisibles.

${ }^{40}$ Bref espace laissé blanc entre deux virgules.

${ }^{41}$ Allusion à l'Institut national genevois créé par James Fazy, que Rifaud mentionne aussi dans son carnet (BGE, Ms. fr. 7879, env. 7, carnet, pp. $24 \mathrm{v}^{\circ}$ à $25 \mathrm{r}^{\circ}$ ).
} 
connus dans la republique des lettres et des $\mid$ sciences pour conjointement avec les savants | et hommes de lettre indigene lui donner un relief | tout europen, etant lui méme de ce nombre, ${ }^{42}$

Les intentions de Rifaud sont on ne peut plus claires. Fort de son expérience d'explorateur de l'Égypte, se référant toujours à une œuvre en devenir pour laquelle il possède quelques souscripteurs, il se présente en observateur privilégié des mœurs et coutumes et souhaite produire un ouvrage original sur Genève (ou la Suisse, le texte reste ambigu). Entreprise ambitieuse, puisqu'il entend même s'adresser aux « indigènes » qui, grâce à cette « recherche » connaîtront mieux leur pays, voire leur identité... Voilà qui explique incontestablement l'abondance de documents que le voyageur a également réunis sur Venise, la Hollande, la Belgique, la Suisse (et finalement Genève). Reste toutefois à tenter d'évaluer l'apport de Rifaud à cette anthropologie européenne.

Un point interpelle pourtant. James Fazy n'est certainement pas un moindre quidam. Après avoir milité contre le gouvernement aristocratique et conservateur, parfois violemment, il est le principal rédacteur de la Constitution genevoise de 1847 et effectivement le fondateur de l'Institut national genevois (1853), dont Rifaud nous annonce la création «en primeur». Avec la démolition des fortifications, c'est Fazy qui modèlera le visage de la Genève moderne, comme il sera l'un des artisans principaux de la démocratisation de l'enseignement ${ }^{43}$ ou du développement de nombreuses institutions sociales. Fazy est donc une personnalité engagée, dont on imagine un emploi du temps a priori chargé. Or il trouve la liberté d'accorder à Rifaud trois entretiens en moins d'une dizaine de jours : on s'étonne d'une telle facilité d'accès. Se seraient-ils connus à Paris, où Fazy résida par intermittence jusqu'en 1833 ? Est-ce par l'intermédiaire de cercles maçonniques, dont Rifaud se déclare ouvertement adhérent et dont on sait Fazy très proche ${ }^{44}$ ?

\footnotetext{
${ }^{42}$ BGE, Ms. fr. 7879, env. 7, $\mathrm{f}^{\circ} 7 \mathrm{r}^{\circ}$ et $\mathrm{v}^{\circ}$.

${ }^{43}$ Enseignement sur la qualité duquel Rifaud exprime bien des doutes, même s'il reconnaît qu'il est accessible au plus grand nombre (BGE, Ms. fr. 7879, env. 7, carnet, p. $22 \mathrm{r}^{\circ}$ et $\mathrm{v}^{\circ} ; \mathrm{f}^{\circ} 14 \mathrm{r}^{\circ}, \mathrm{f}^{\circ} 18$ $\mathrm{r}^{\circ}$ et $\left.\mathrm{v}^{\circ}, \mathrm{f}^{\circ} 46 \mathrm{r}^{\circ}\right)$.

44 À Paris, Fazy avait rejoint la « Charbonnerie » et le cercle « Aide-toi le ciel t'aidera », dont les idéaux ne sont guère éloignés de la maçonnerie (Dictionnaire historique de la Suisse, article en ligne de J. DE SENARCLENS, consulté le 30 août 2021). On signalera les relations suivies entretenues par Fazy avec Louis-Napoléon Bonaparte dès 1835-1836 (date de sa tentative de coup d'état à Strasbourg). Voir infra, p. 27.
} 


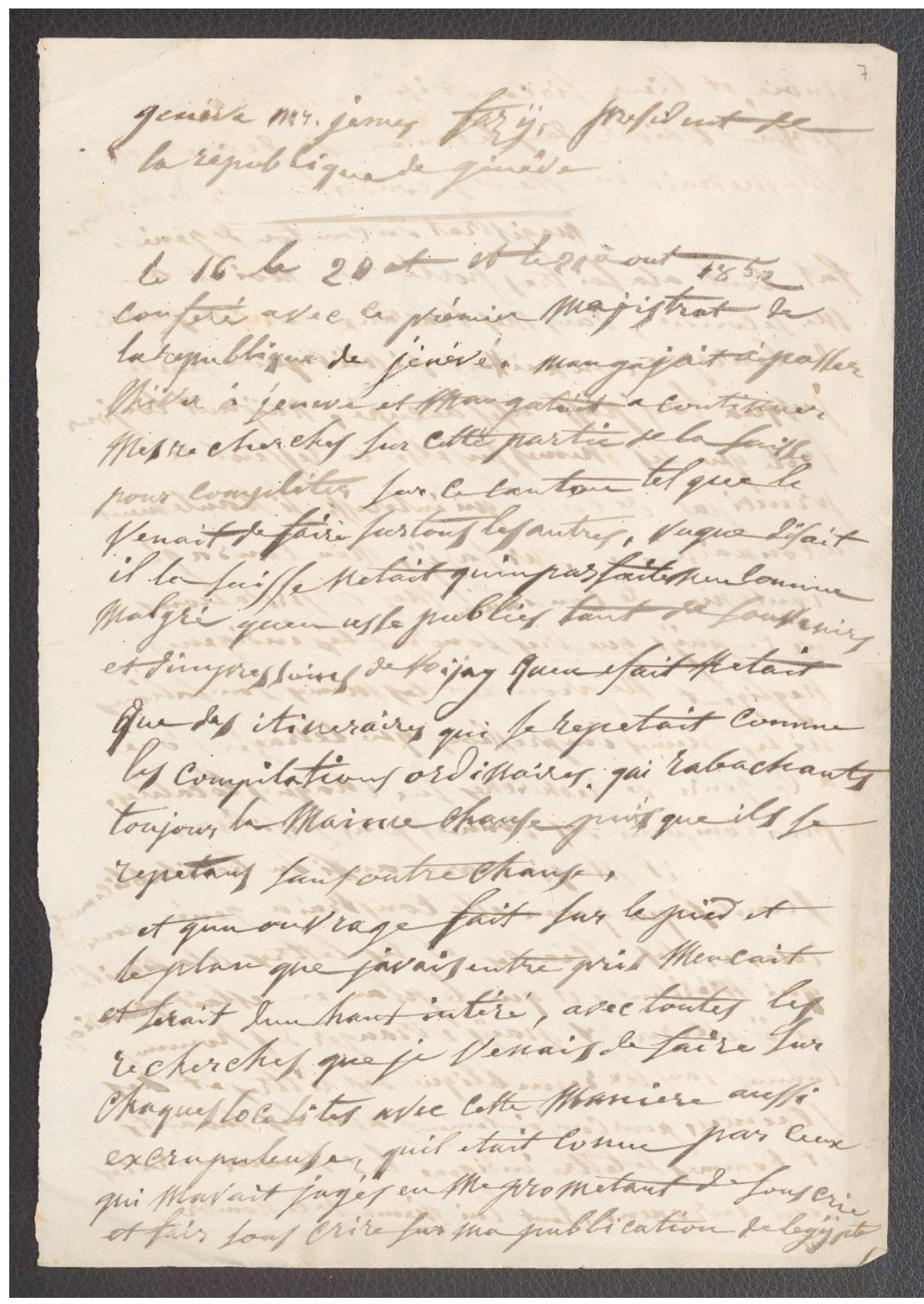

Fig. 2 : Notes manuscrites relatant les entretiens que Rifaud eut avec James Fazy en août 1852 (C Bibliothèque de Genève, Ms. fr. 7879/7, $\mathrm{f}^{\circ} 7 \mathrm{r}^{\circ}$ ) 


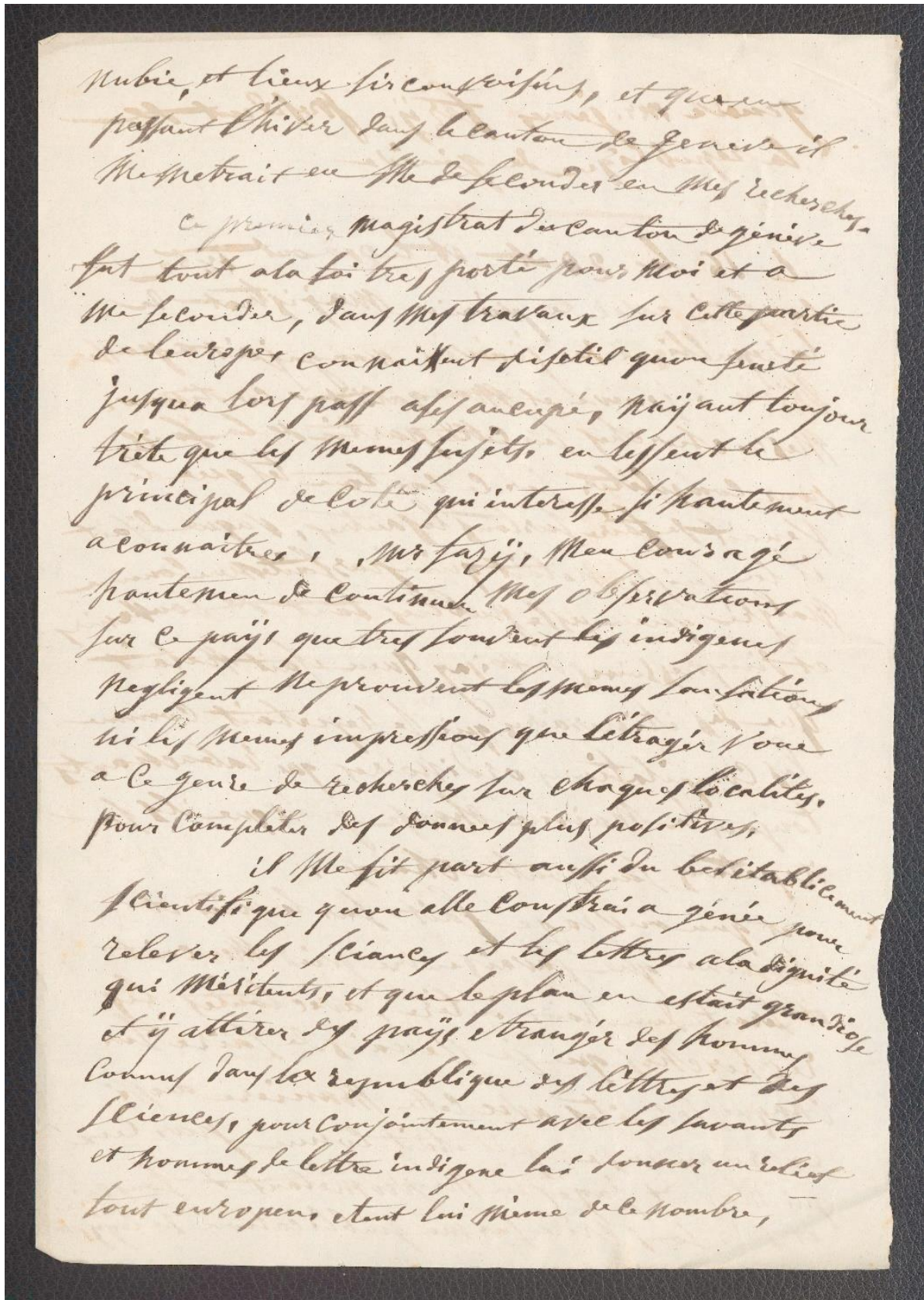

Fig. 3 : Notes manuscrites relatant les entretiens que Rifaud eut avec James Fazy en août 1852 (C Bibliothèque de Genève, Ms. fr. 7879/7, $\mathrm{f}^{\circ} 7 \mathrm{v}^{\circ}$ ) 
La première impression qui se dégage des feuillets consacrés à Genève est positive. La topographie, les transformations urbaines que vit alors la cité, les promenades dans ou autour de la ville, la vie culturelle : tout est conforme à ce que nous enseigne l'histoire genevoise du milieu du XIX ${ }^{\mathrm{e}}$ siècle. Un esprit critique relèvera peut-être que la statue de Jean-Jacques Rousseau, érigée sur l'île tardivement baptisée au nom de cet esprit des Lumières, est attribuée fort correctement à James Pradier (1790-1852), mais que Rifaud la date tour à tour de 1833 , puis de $1834^{45}$. Curieusement, il la qualifie de statue « équestre », probable confusion de vocabulaire, puisque Rousseau y est représenté "à l'Antique » et non perché sur un quadrupède. Sourire sans doute lorsqu'il nous dit que le philosophe genevois aimait à se retirer sur cette île (dont il nous précise fort correctement qu' au XVIII" siècle elle s'appelait encore « Île aux Barques » et était un vestige des fortifications) pour y méditer et y écrire ses réflexions. À l'évidence, Rifaud n'a pas lu les Confessions, et ne connaît l'œuvre de Rousseau que par ouï-dire. Il y détaille en revanche les bancs mis à disposition des passants ou les concerts qui s'y donnent l'été, d'autant plus qu'il y venait sans aucun doute en voisin, logeant à quelques centaines de mètres de cet agrément public. Ce sont là les rares approximations de Rifaud, lorsqu'il décrit la ville.

Même s'il prétend s'affranchir des guides «touristiques » qui l'ont précédé, Rifaud s'en inspire largement ${ }^{46}$. Il en résume de larges extraits, quand il ne les recopie pas tout simplement! Ainsi, ses conseils et ses itinéraires pour se rendre au Salève, jusqu'au Pont de Caille ${ }^{47}$, ou aux Voirons ${ }^{48}$, parcourir les rives du lac,

${ }^{45}$ Sur ce monument et son emplacement : BGE, Ms. fr. 7879, env. 7, carnet, pp. $19 \mathrm{r}^{\circ}$ et $\mathrm{v}^{\circ}, 33$ $\mathrm{v}^{\circ}, 34 \mathrm{r}^{\circ}, 35 \mathrm{v}^{\circ}$ à $36 \mathrm{r}^{\circ}$. La statue fut inaugurée en 1835 (Dictionnaire historique de la Suisse, article en ligne de Cl. LAPAIRE, consulté le 7 septembre 2021).

${ }^{46}$ Guides consultés lors de cette recherche : Guides MANGET : Jacques-Louis MANGET, Manuel topographique et statistique de la Ville et du Canton de Genève, Genève (imprimerie Cherbuliez) 1823 ; Genève. Itinéraire nouveau et complet de cette ville et de ses environs, Genève (imprimerie Combe) 1840 ; Guide du voyageur autour du lac de Genève, Genève (imprimerie Gruaz) 1850. Guides AUdin-RICHARD : RICHARD, Guide du voyageur en Suisse, Paris (librairie Audin-Canel) 1824, pp. 1-27 ; EBEL \& LUTZ, revu et augmenté par RICHARD, Manuel du voyageur en Suisse, Paris (librairie Audin) 1836, pp. 1-24 ; Jean-Marie-Vincent AUDIN \& Johann Gottfried EBEL, Manuel du voyageur en Suisse (revu d'après Murray), Paris (Guides de Richard) 1843, pp. 33-76. Guides MurRaY : Murray, Manuel du voyageur en Suisse, Paris (L. Maison, éditeur des Guides de Richard) 1844, pp. 222-240. Guides JoANNE : Adolphe JOANNE, Itinéraire descriptif et historique de la Suisse, Paris-Leipzig (imprimerie Renouard) 1841, pp. 31-33 et 139-154 ; Adolphe JoAnNE, Itinéraire descriptif et historique de la Suisse, Paris (Maison, éditeur des Guides de Richard) 1853, pp. 44 et $96-110$.

Les emprunts recopiés par Rifaud se signalent également par une syntaxe ou une orthographe moins « personnelles ».

${ }^{47}$ BGE, Ms. fr. 7879, env. 7, carnet, p. $14 v^{\circ} ; f^{\circ} 41 r^{\circ}$ à $44 r^{\circ}$.

${ }^{48}$ BGE, Ms. fr. 7879, f $\mathrm{f}^{\circ} 37 \mathrm{r}^{\circ}$ à $38 \mathrm{v}^{\circ}$. 
pour exacts qu'ils soient ${ }^{49}$, n'en sont pas moins des resucées des recommandations de Audin-Richard, Murray, Joanne, Manget, qui eux-mêmes s'empruntaient de larges portions de texte! On ne trouve dans les papiers Rifaud nulle description de la ville ou de ses alentours qui ne soient déjà commentée dans les publications précitées : au mieux y aura-t-il ajouté la présence de quelques bancs publics ou décompté quelques arbres ${ }^{50}$. Rifaud emprunte également à ces sources des renseignements sur le salaire des Conseillers d'État ou sur la formation du contingent genevois, l'industrie, le nombre de personnes actives dans l'horlogerie $^{51}$. Sa prose n'est au mieux que variantes.

La description des monuments ou des institutions est également une succession d'emprunts. Comme beaucoup de visiteurs, il mentionne la rampe qui donne accès aux différents étages de l'Hôtel-de-Ville (tour Baudet) et la possibilité de s'y rendre avec une petite voiture (Rifaud dut d'ailleurs y déposer ses papiers, l'expérience a donc été vécue) $)^{52}$. Sa présentation de la Cathédrale Saint-Pierre est relativement détaillée ${ }^{53}$, avec non seulement des précisions glanées dans les guides touristiques, mais également extraites de publications de Jean-Daniel Blavignac qu'il pourrait avoir rencontré personnellement ${ }^{54}$. Rifaud mentionne sommai-

\footnotetext{
${ }^{49}$ Même si parfois confus, ou trop rapidement résumés, ce qui permet de douter qu'il n'ait jamais suivi ces itinéraires. Un bon exemple est sa description d'une longue promenade sur la rive droite du lac (BGE, Ms. fr.7879, env. 7, $\mathrm{f}^{\circ}$ 27-28), remontant de village en village de Genève jusqu'à Céligny, où Rifaud enchaîne, à l'intérieur du même paragraphe, par l'expression « a peu de distance on visite » $\left(\mathrm{f}^{\circ} 27 \mathrm{r}^{\circ}\right)$, qui ferait revenir le promeneur sur ses pas de plus de vingt kilomètres !

Cette excursion se termine à Ferney, où Rifaud affirme que « dans le parc on | vous montre le grand orme qui fut planté par | voltaire et de ses propres mains en $1824<s i c>$ sur le|quel on voit des traces de la foudre $»\left(\mathrm{f}^{\circ} 28 \mathrm{v}^{\circ}\right)$, amalgamant une information du guide Manget de 1840 (p. 89), qui signalait que l'arbre planté par Voltaire en personne avait été foudroyé en 1824 !

${ }^{50}$ Sur la topographie de la ville et du canton : BGE, Ms. fr. 7879, env. 7, carnet, pp. $34 \mathrm{v}^{\circ}$ à 35 $\mathrm{v}^{\circ}, 38 \mathrm{r}^{\circ}$ à $40 \mathrm{v}^{\circ} ; \mathrm{f}^{\circ} 3 \mathrm{r}^{\circ}, \mathrm{f}^{\circ} 17 \mathrm{r}^{\circ}$ et $\mathrm{v}^{\circ}, \mathrm{f}^{\circ} 20 \mathrm{r}^{\circ}$ à $21 \mathrm{v}^{\circ}, \mathrm{f}^{\circ} 26 \mathrm{r}^{\circ}, \mathrm{f}^{\circ} 45 \mathrm{r}^{\circ}$. Promenades à l'intérieur de la ville, paysage et parcs : BGE, Ms. fr. 7879 , env. 7, carnet, pp. $8 \mathrm{v}^{\circ}, 11 \mathrm{v}^{\circ}$ à $13 \mathrm{r}^{\circ}, 15 \mathrm{r}^{\circ}$ à $16 \mathrm{r}^{\circ}$, $19 \mathrm{r}^{\circ}$ et $\mathrm{v}^{\circ}, 27 \mathrm{v}^{\circ}, 38 \mathrm{r}^{\circ} ; \mathrm{f}^{\circ} 24 \mathrm{r}^{\circ}$ et $\mathrm{v}^{\circ}$. Évoquant la météorologie, Rifaud se plaint même de la rigueur du climat hivernal, alors qu'il n'a résidé dans la cité qu'en juillet et août : BGE, Ms. fr. 7879, carnet, pp. $4 \mathrm{v}^{\circ}, 36 \mathrm{v}^{\circ}$; fo $10 \mathrm{r}^{\circ}$ et $\mathrm{v}^{\circ}, \mathrm{f}^{\circ} 13 \mathrm{r}^{\circ}, \mathrm{f}^{\circ} 22 \mathrm{r}^{\circ}$ et $\mathrm{v}^{\circ}$.

${ }^{51}$ BGE, Ms. fr. 7879, env. 7, fo $45 \mathrm{v}^{\circ}$ et $46 \mathrm{v}^{\circ}$. (source : guide JOANNE 1841, p. 147).

${ }^{52}$ Ms. fr. 7879, env. 7, carnet, pp. $25 \mathrm{v}^{\circ}$ à $27 \mathrm{r}^{\circ}, 29 \mathrm{r}^{\circ}$; $\mathrm{f}^{\circ} 31$. Rifaud décrit s'être rendu auprès de l'office des passeports en apportant avec lui l'ensemble des firmans et autres attestations en arabe, russe et autres langues qu'il avait accumulés lors de ses pérégrinations, pour épater les préposés à l'enregistrement de son séjour.

${ }^{53}$ BGE, Ms. fr. $7879, \mathrm{f}^{\circ} 47 \mathrm{r}^{\circ}$ et $\mathrm{v}^{\circ}$ et $48 \mathrm{r}^{\circ}$.

${ }^{54}$ Le conditionnel est de mise. L'archéologie est un premier point commun qui peut les avoir réunis, comme l'attirance de Blavignac pour la maçonnerie (il ne semble toutefois pas y avoir adhéré avant 1854).

L'architecte et archéologue Jean-Daniel Blavignac (1817-1876) dirigeait alors la restauration de la cathédrale (Dictionnaire historique de la Suisse, article en ligne de A. BRULHART, consulté le 30 août 2021). Il publie notamment : Description monumentale de l'église de Saint-Pierre, ancienne
} 
rement les principales bibliothèques, l'Arsenal, le Musée Rath, les collections privées (autographes et numismatique de MM. Coindet et Marin, bibliothèque de M. Favre au parc de la Grange), sans qu'on sache s'il les a fréquentées ${ }^{55}$. Plus intéressante sous la plume d'un ancien découvreur de l'Égypte ancienne, sa description du Musée Académique : «on visite aussi près de la grande rue | le musée académique, et le cabinet dhistoire | naturelle ou sont aussi des collections géologiques, | et de mineraux de la Suisse ainsi que des | poissons des lacs de la Suisse, un elephant. || On a ensuite la salle des antiquites | et de medailles ou se trouve aussi une momie $\mid$ et de petites antiquites egÿptiennes, etc $\mid$ et puis on passe ala colection d'anatomie et de $\mid$ dela au laboratoire de chimie, et le cabinet de phÿsique. || On trouve dans le conservatoire un grand nombre | d'herbiers $»^{56}$. Le lecteur reste étonné de la placidité de Rifaud face à la petite collection égyptienne alors présentée, puisqu'il pourrait être à l'origine de la découverte de certaines pièces, ou que celles-ci auraient pu lui évoquer maints souvenirs ${ }^{57}$. Il est vrai qu'aucune œuvre présentée n'avait l'ampleur des imposantes et lourdes rondesbosses rapportées en Europe avec les collections Drovetti.

Mais il y a, à côté de ces emprunts, des observations plus personnelles, qui sont peut-être celles auxquelles Rifaud fait référence lors de son entretien avec James Fazy. Elles nous étonnent tant par leur naïveté, leurs contradictions que par la situation désespérée qu'elles laissent transparaître de leur auteur.

Rifaud loge dans un quartier récemment édifié à la suite de l'abandon des fortifications de la ville. Ces nouvelles constructions, qui s'étendent tout autour de l'ancienne cité, sont largement mentionnées dans les relations de ses promenades, qualifiées de « jolies » ou « belles maisons », dont il compte avec attention

cathédrale de Genève, Genève (chez Julien) 1845 ; « Notes historiques sur l'église de Saint-Pierre ancienne cathédrale de Genève », Société d'Histoire et d'Archéologie, Genève 1849, pp. 25-70 ; «Restauration des stalles hautes de l'ancienne cathédrale de Genève », Société d'Histoire et d'Archéologie (?), Genève 1849, pp. 73-83; Rapport sur les recherches et travaux exécutés en 1850 dans le temple de St-Pierre, Genève (imprimerie Élie Carey) 1850.

${ }^{55}$ BGE, Ms. fr, 7879, env. 7, $\mathrm{f}^{\circ} 23 \mathrm{v}^{\circ}$. Collections notamment référencées dans les guides AUDINRICHARD 1836 (p. 8) et AUDIN RICHARD 1843 (p. 38).

${ }^{56}$ BGE, Ms. fr. 7879, env. 7, fo 32 r .

${ }^{57}$ Aux guides consultés (AUDIN-Richard 1836, p. 6 ; AUdin-RiCHARD 1843, p. 39, qui la qualifient de «très belle »; JOANNE 1853, p. 104) mentionnant une momie provenant de Thèbes (corps démailloté de la dame Tjesmoutpert, aujourd'hui conservé au Musée d'art et d'histoire, inv. D 242), Rifaud ajoute la précision (BGE, Ms. fr. 7879, env. 7, $\mathrm{f}^{\circ} 32 \mathrm{r}^{\circ}$ ) « et de petites antiquites egÿptiennes » (environ deux cents pièces). Sur la constitution de la collection genevoise et l'apport du consul Drovetti, voir J.-L. ChAPPAZ, «La diligence, l'autruche et la momie empaillée. Aux origines de la collection d'antiquités égyptiennes », dans J.-L. ChAPPAZ \& Cl. RiTsChARD (éds), Voyages en Égypte, Genève 2003, pp. 98-117. 
les étages ${ }^{58}$. À nul moment cependant, il ne relève l'originalité et la caractéristique des immeubles qui l'entourent. Leur dernier étage voit se démultiplier le nombre de fenêtres et abrite les ateliers des artisans (« cabinotiers ») spécialisés dans les métiers de l'horlogerie, qui bénéficiaient ainsi de davantage de lumière naturelle pour exercer leur activité (horlogers, boîtiers, émailleurs, etc.). Ce manque de curiosité interpelle de la part d'un observateur qui prétend faire œuvre novatrice.

La machine hydraulique construite par l'ingénieur Abeille pour alimenter en eau toute la cité force sa curiosité, son admiration et des commentaires développés sur son débit et le diamètre des tuyaux (réminiscence peut-être des difficultés d'approvisionnement vécues lors de ses excursions dans les déserts) $)^{59}$.

Évoquant rapidement l'agriculture, dont il juge les exploitations fort mal développées, il apprécie la qualité des cépages d'une vigne ${ }^{60}$, puis déclare tous les vins produits dans la campagne genevoise trop acides, « au point quon ne peut le boire sans eaux $»^{61}$.

De la population, Rifaud note qu' « on ne | trouve pas moins chez les | génévois de tres belles tailles | qui sont bien au dessus de la | moÿene ou onÿ observe ausssi | une phÿsionomie inteligente | et lenez grec, on trouve | aussi dans son regard quelque $\mid$ chause de refleechi ou meme $\mid$ quelque chause de malin $\mid$ on trouve aussi de grandes $\mid<\mathrm{f}^{\circ} 19>$ tailles chez les genevoises et $\mid$ meme un beau sang, mais $\mid$ oni observe un peu de raideur $\mid$ on trouve aussi chéz le genevoi | delactivite et perséverent $\mid$ dans les affaire, le genevoi | est au prémier abord réservé $\mid$ et tres porte pour les voÿages $\mid$ pour le commerce $<\ldots>\mid$ les femmes ÿ sont $\mid$ bien instruites elles sont pieuses $\mid$ et bonnes menagères $\rangle^{62}$. Portrait physique et propos général relativement neutre, qui tranchent radicalement avec une multitude d'autres appréciations beaucoup moins flatteuses. Le français parlé à Genève est truffé d'« intonations nassales gutturales $\mid<$ verso : > trainante ou il ÿ a dela | monotonie dans les inflexions | ainsi que dans la forme des phrase » et on entend dans les campagnes une grande variété de patois ${ }^{63}$. Mais surtout, les Genevois sont bruyants, parlent tous à la fois sans s'écouter ${ }^{64}$. La vie y est chère, particulièrement

\footnotetext{
${ }^{58}$ Notamment BGE, Ms. fr. 7879, env. 7, carnet, pp. $1 \mathrm{v}^{\circ}, 4 \mathrm{r}^{\circ}$ et $\mathrm{v}^{\circ}, 36 \mathrm{r}^{\circ}, 37 \mathrm{v}^{\circ} ; \mathrm{f}^{\circ} 8 \mathrm{r}^{\circ}, \mathrm{f}^{\circ} 17$ $\mathrm{r}^{\circ}$ et $47 \mathrm{r}^{\circ}$.

${ }^{59}$ BGE, Ms. fr. 7879, env. 7, carnet, p. $13 \mathrm{r}^{\circ}$ à $14 \mathrm{r}^{\circ}, 18 \mathrm{r}^{\circ}$; $\mathrm{f}^{\circ} 11 \mathrm{r}^{\circ}$ et $\mathrm{f}^{\circ} 32 \mathrm{v}^{\circ}$. Les guides touristiques la mentionnent également, mais de façon plus discrète. Les manuscrits de Rifaud laissent toutefois le nom de l'ingénieur en blanc (carnet, p. $14 \mathrm{r}^{\circ}$ ).

${ }^{60}$ BGE, Ms. fr. 7879, env. 7, f $5 \mathrm{v}^{\circ}$.

${ }^{61}$ BGE, Ms. fr. 7879, env. 7, $\mathrm{f}^{\circ} 8 \mathrm{v}^{\circ}$.

${ }^{62}$ BGE, Ms. fr. 7879 , env. 7 , fo $18 \mathrm{v}^{\circ}$ et $19 \mathrm{r}^{\circ}$.

${ }^{63}$ BGE, MS. fr. 7879, env. 7, fo $19 \mathrm{r}^{\circ}$ et $\mathrm{v}^{\circ}$; voir aussi carnet, p. $28 \mathrm{v}^{\circ}$.

${ }^{64}$ BGE, MS. fr. 7879, env. 7, carnet, p. $16 \mathrm{v}^{\circ}$.
} 
les logements, et les habitants abusent des étrangers, promettant monts et merveilles sans tenir parole, sont roublards et peu fiables ${ }^{65}$, sous le prétexte que la saison «touristique » est réduite à quatre mois durant lesquels ils doivent constituer leur revenu annuel. Comme tous les Suisses, ils sont avares, inhospitaliers et égoïstes ${ }^{66}$, sans commisération pour leur prochain. La débauche les guette :« si lon escrute le genre de vie des mainages | des genevois on trouve sont tres peux aupres de | leur familles et quils preferent rester jusqu'à 11 heur $\mid$ et minuit dans les lieux publics pour boire et $\mid$ causer tandis que la femme atand inutilement $»^{67}$. Ce qui s'exprime aussi par ces propos : « il est plus porte pour la bouteille que pour la $\mid$ parole, et lhoneur ne tient pas à couer $\left\langle\right.$ ? $>^{68}$, \| cette differencee de caractere fait tout leur $\mid$ leur $\langle$ sic $>$ malleur quoi que celui de interes soit le | premier dans leurs manière de penser comme celle $\mid$ dagir ${ }^{69}$; " en resumé les suisses français cenesont que des $\mid$ allemands afranchise ou des savoÿards dégénerés | etant place entre la France et la savoi, | cest se qon peut dire nitoi nimoi, cest $\mid$ adire nilun nilautre $»^{70}$.

Quant aux femmes, beaucoup seraient - comme les hommes - édentées dès leur plus jeune âge en raison du climat et de la forte bise ${ }^{71}$. Oubliées de leurs maris, elles ne répugnent pas le vin et le supportent assez bien ${ }^{72}$. Portées sur l'orgueil de la toilette et l'appât de l'intérêt, trompeuses et fausses, rouées envers les étrangers, débauchées, intrigantes et enclines au libertinage, elles laissent se répandre des 《 maladies secretes (...) à des ages tres pres matures $»^{73}$. Rifaud leur concède que « quoi quases de|pravees elles aurait plus de constances que les | hommes elles sont pour une grande partie plus | sensibles en vers les malheureux $»^{74}$. Elles compatissent plus volontiers aux malheurs ou aux difficultés des étrangers, apprécient les Français en avouant l'indifférence et la brutalité des hommes suisses à leur égard ${ }^{75}$.

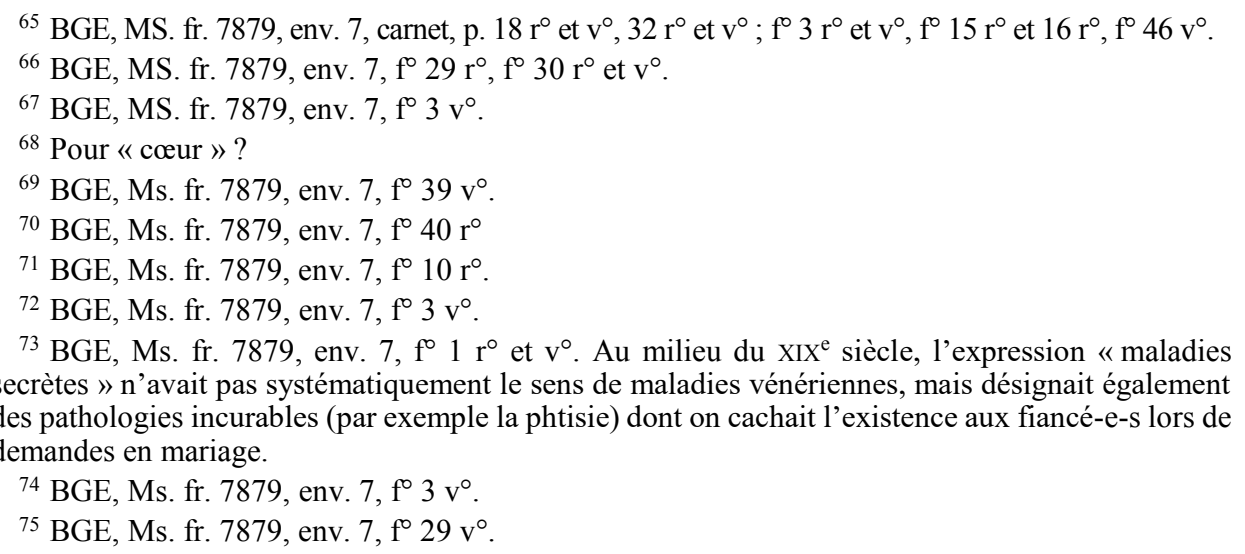


Ces appréciations, guère nuancées, trouvent sans doute une large part d'explication dans l'attitude et la situation de Rifaud lui-même. Il se présente comme un érudit voyageur, fier de son grade de chevalier de la légion d'honneur, sans aucun doute imbu de sa personne et de l'œuvre qu'il porte en lui, mais fatalement désargenté. Il est probable que ses interlocuteurs l'auront pris pour ce qu'il voulait paraître et non pour ce qu'il était ${ }^{76}$. On lit sous sa plume des rancunes similaires à l'encontre des populations de Belgique et de Hollande ${ }^{77}$.

Si ses notes et propos ne manquent ni de contradictions, ni par moment d'une amertume profonde qui laisse entendre une personnalité fragile, si ce n'est bipolaire et malade, on retrouve également dans les pages que Rifaud consacre à Genève l'indignation qui était la sienne lorsqu'il commentait les cruelles exactions dont les paysans égyptiens étaient les victimes de la part du gouvernement de Méhémet-Ali. Si sa dénonciation de l'égoïsme et du manque de compassion des Suisses envers les étrangers en apporte sans doute un premier rappel (quoique probablement très personnel), une autre injustice le révolte. Le mépris dont les catholiques sont l'objet de la part des populations réformées non seulement à Genève, mais également à Bâle, où les situations sont mises en parallèles. Les protestants « accaparent» temples et anciennes églises. Ne laissant aux catholiques, qui représentent au moins le tiers de la population, qu'une église trop étroite, obligeant nombre d'entre eux à suivre les messes sur le parvis. Et alors que des édifices se construisent pour les autres religions en fonction de la place libérée par la démolition des remparts de la ville ${ }^{78}$, mille obstacles paraissent

\footnotetext{
${ }^{76}$ En témoigne une brève nécrologie publiée par le Journal de Genève (12 septembre 1852) : « Jeudi, jour du Jeûne genevois, est décédé subitement à Genève, M. le chevalier J.-J. Rifaud de Marseille, chevalier de la Légion d'honneur, et membre de plusieurs académies ou sociétés savantes de France, d'Angleterre, de Suède et d'autres pays.

» M. Rifaud publiait en ce moment un magnifique ouvrage sur l'Egypte, la Nubie et les pays circonvoisins.

» Il désirait passer l'hiver à Genève, où il aurait probablement fait part à nos concitoyens, dans quelques cours publics, des connaissances variées qu'il avait acquises dans vingt sept années de voyage.

» La police a fait mettre immédiatement les scellés sur les manuscrits et les dessins qui emplissaient la chambre de ce voyageur. »

Seul objet de valeur retrouvé dans sa chambre : une montre en or (communication de Roger Rosset).

${ }^{77}$ BGE, Ms. fr. 7877,7878 et 7878 bis, passim.

${ }^{78}$ On rappellera qu'à Genève l'attribution des terrains résultant de la démolition des fortifications à quelques communautés religieuses permit l'édification de l'église orthodoxe, de l'église anglicane, de la basilique Notre-Dame (catholique), de la synagogue et du Sacré-Cœur, à l'origine temple maçonnique. En 1852, seule l'église de Saint-Germain (dans laquelle prêcha naguère Calvin !) relevait du clergé catholique.
} 
alors dressés pour ralentir le chantier de la basilique Notre-Dame qui ne verra effectivement sa consécration que plus tardivement. Rifaud n'a pas de mots assez forts pour dénoncer cet abus et ce manque de considération envers la foi qui est la sienne $^{79}$.

Avec une même ardeur, il plaindra également les invités des cantons extérieurs à une première fête fédérale de gymnastique proposée par les Genevois en juillet 1852. Ils auront été mal accueillis, mal logés, exclus du bal, en quelque sorte « rançonnés » par les organisateurs qui n'auraient cherché, dénonce-t-il, qu'à s'enrichir sur leur $\operatorname{dos}^{80}$ !

\section{Un entretien avec le général Guillaume-Henri Dufour (1787-1875)}

Rifaud sait aussi se montrer affable et jouer de ses relations. Comme il l'a fait à la fin du mois d'août 1852 auprès de James Fazy, il avait obtenu en juillet un entretien avec une autre personnalité genevoise : le général Guillaume-Henri Dufour (voir compte rendu de Rifaud en annexe ${ }^{81}$ ). La biographie du général est intense $^{82}$ et difficile à résumer en quelques lignes. Sa scolarité se déroule alors que la cité, annexée par la France en 1798, était le chef-lieu du Département du Léman. Ce qui lui permettra d'être admis à Polytechnique, d'où il sortira officier, avant de démissionner de l'armée française en 1817, après le retour à la souveraineté nationale. Ingénieur, il contribue à forger le visage de la ville nouvelle, entreprend l'établissement de la carte topographique de la Suisse, et cofonde et dirige également l'école centrale (i. e. : école militaire fédérale) de Thoune en 1819. Il prendra à quatre reprises le commandement suprême de l'armée ${ }^{83}$, notamment lors de la guerre civile dite du Sonderbund (1847), où sa conduite humaine des opérations lui vaudra le surnom de "général pacificateur ». Il sera aussi l'un des initiateurs du Comité international de secours aux blessés (aujourd'hui : Comité International de la Croix-Rouge) en 1863.

Parmi ses élèves de l'école militaire fédérale se trouve un jeune Français exilé en Suisse : Louis-Napoléon Bonaparte (1808-1873), avec lequel Dufour conservera d'excellentes relations après son retour en France et pendant toute sa

\footnotetext{
${ }^{79}$ BGE, Ms. fr. 7879, env. 7, carnet, pp. $5 \mathrm{r}^{\circ}$ à $8 \mathrm{r}^{\circ}, \mathrm{f}^{\circ} 2 \mathrm{r}^{\circ}$ et $\mathrm{v}^{\circ}$ (voir aussi : $\mathrm{f}^{\circ} 8 \mathrm{v}^{\circ}$ et $\mathrm{f}^{\circ} 18 \mathrm{r}^{\circ}$, rappelant l'importance du nombre de catholiques dans la population).

${ }^{80}$ BGE, Ms. fr. 7879, env. 7, $\mathrm{f}^{\circ} 4 \mathrm{r}^{\circ}$ et $\mathrm{v}^{\circ}, \mathrm{f}^{\circ} 35 \mathrm{r}^{\circ}$ à $36 \mathrm{v}^{\circ}$.

${ }^{81}$ BGE, Ms. fr. 7879, env. 7, $\mathrm{f}^{\circ} 49 \mathrm{r}^{\circ}$ à $50 \mathrm{v}^{\circ}$.

82 Dictionnaire historique de la Suisse en ligne, article de Jean-Jacques LANGENDORF (consulté le 5 septembre 2021). Voir aussi le catalogue commun des deux expositions G. H. Dufour. L'homme, l'œuvre, la légende / Le portrait topographique de la Suisse (Genève, Maison Tavel, 5 septembre 1987 - 13 mars 1988 et Musée de Carouge, 5 septembre - 13 décembre 1987), Carouge 1987.

${ }^{83}$ En $1847,1849,1856$ et 1859.
} 
carrière politique ${ }^{84}$. Élu président de la République en 1848 , le "princeprésident » est proclamé empereur en 1852 sous le nom de Napoléon III.

C'est bien cette relation privilégiée qui motive Rifaud. Le seul point commun entre les deux hommes, excepté l'intérêt possible du général pour les travaux de Rifaud, réside dans le fait qu'ils arborent tous deux une légion d'honneur. Toutefois, Dufour refuse poliment de recommander Rifaud et l'édition de son œuvre au prince président, ce qui suscite naturellement l'amertume profonde de l'explorateur (voir annexe) et son dégoût du genre humain. Il est vrai que, imparfaitement renseigné, il a cru que son interlocuteur était un général français et s'est trouvé étonné de ne pas avoir été traité en compatriote. Il n'avait qu'à moitié tort, mais ce détail prouve aussi son impréparation et sa difficulté à se renseigner diplomatiquement et avec subtilité au-delà de ses premières intuitions.

$\mathrm{Au}$ terme de cette rapide présentation des impressions genevoises laissées par Rifaud, il convient de s'interroger si sa "méthode", transposée à sa documentation égyptienne, peut être source d'une information originale concernant l'histoire de ce pays. Force est d'en douter. Certes, dans la première moitié du $\mathrm{XIX}^{\mathrm{e}}$ siècle, les commentaires extérieurs à propos de l'Égypte sont moins nombreux que les publications sur Genève accessibles en 1852, mais il y a tout lieu de penser que Rifaud aura également profité de récits récents lors de la rédaction des commentaires à son Voyage, ou qu'il aura recueilli des témoignages oraux de personnalités qu'il aura su aborder et rencontrer ${ }^{85}$. Ses états d'âme, ses emportements face à ce qu'il considère comme des injustices, le conduisent à des exagérations, et surtout à des propos très superficiels, sans réel examen des causes, voire de la relativité des faits ${ }^{86}$. En revanche, il sait parfaitement s'introduire ou être introduit auprès de personnalités dont il espère qu'elles encourageront les souscriptions à ses volumes ou le recommanderont auprès de mécènes ou de décideurs culturels. Reste ouverte la question de savoir pourquoi il n'en retira que des refus, ou presque.

\footnotetext{
${ }^{84}$ Louis-Napoléon Bonaparte fréquenta l'école centrale de Thoune de 1830 à 1836.

${ }^{85}$ Il rencontre même « Champolliont » en 1832, peu de jours avant son décès. L'entretien tournera court (BGE, Ms. fr. suppl. 113, $\mathrm{f}^{\circ} 139$ ).

${ }^{86}$ À sa lecture, on comprend, voire on partage, le dégoût que lui inspire les (ex)actions commises au nom du gouvernement de Méhémet-Ali. Rien n'est pourtant dit sur les objectifs et l'ambition du vice-roi à « moderniser » son royaume.
} 


\section{Annexe : Visite au général Guillaume-Henri Dufour}

Le 27 juillet 1852, soit à la veille de la première fête fédérale de gymnastique présidée notamment par le général Dufour, Rifaud obtient un rendez-vous au cours duquel il sollicite de son interlocuteur une recommandation auprès du prince président Louis-Napoléon Bonaparte. Dufour l'éconduit poliment. Perdant peutêtre l'un de ses derniers espoirs de soutien, Rifaud couche sur le papier une relation de cette rencontre (Ms. fr. 7879, env. 7, $\mathrm{f}^{\circ} 49 \mathrm{r}^{\circ}$ à $50 \mathrm{v}^{\circ}$ ), d'une rédaction particulièrement hâtive, mêlant le compte rendu des discussions à sa déception et son amertume profonde. Une tentative de donner à ce texte - par moment déroutant - un certain suivi est proposée à la suite de la retranscription du texte manuscrit.

« le 27 juillet 1852 géneve

» la sivilisation diton elle à fait des grands | progres depuis lage du barbarisme, mais aussi | elle a tres agrandi les progres du barbarisme | légoisme, a un sublime dégré, comme il | ÿ at tant de fauses reputation exurpée je | voulu connaitre si celle que les Suisses accordait | au général du four elle était bien meritée | sur se qui consernait son esprit national et $\mid$ de compatriotisme dont on me louetans $<$ ? $>^{87}$ ainsi $\mid$ que son extreme obligence, pour ses compatriotes | qui sertenes sirconstance avait recours alui $\mid$ dans son nouveau paÿs adoptif en vous le | citant come un home bons obligent et $\mid$ surtout tres pieux, etct.

» apres mes 47 annees de voÿages en afrique | asie et leurope an juillet 1852 metrouvent | randus a génève ou se terminait mes $47 \mid$ annees de voÿages, dapres les conseils que | lon me donnait je voulus aussi rendre ma visite | a ce veteran de larmée française et me trouvant $\mid$ en conferance avec son excellence tout en tetete|atete dans son cabinet, lui faisent part de $\mid$ mon arrivee et de mes longs voÿages, je le $\mid$ pries sil pouvait mobligé den faire part ason tour $<$ ? $>^{88} \mid$ (verso :) president de la republique française apres | quil aura visite cet amas de trvail $<s i c\rangle$ sur les trois $\mid$ parties du monde ou ji avait consacré la $\mid$ plus grande partie de ma vie pour ÿ rasemblés $\mid$ tant de materiaux fait $<$ ? $>$ en dessins quan manuscrits $\mid$ desireu de rentrer en france.

» En cette demande le général du four ce retrancét $\langle$ sic $\rangle \mid$ derriere la baricade de ses phrases j'ai tant | pris sur moi en vers le president quau jour

\footnotetext{
${ }^{87}$ Pour « on me louait tant »?

${ }^{88}$ Lecture très hypothétique d'un mot de trois ou quatre lettres (« cher » serait possible, mais moins probable).
} 
d'hui | je monabstient autant que pocible de lui ecrire | et de lui demander quelque chause en faveur des $\mid$ sciences des lettres et beaux arts. vu que tout $\mid$ lemonde se tournait vers lui pour aitre obliges | mais que jaites asses connus des savants et $\mid$ des academies de france pour obtenir se que $\mid$ je desirait et que je devéz leur ecrire directemen | la je vis avec qui javais affaire je repondit | comme lui, ou du moins dans le meme sans et $\mid$ je me disait cest asses causes je vois avec qui jai | affaire malgres que les Suisse musse dit que le général | avait boucoup $<s i c>$ acquis depuis quil etudiet quoi quarrive $\mid$ a lage de 70 ans ${ }^{89}$. mais de je nesavait encore dans $\mid$ quelle partie.

» notre conferance dura 3 quart deheures (?) apres $\mid$ je pris conjét de son exellence suissises $<$ ? $>$ et je | le quité au pas de la porte de son jardin ou il | $<\mathrm{f}^{\circ} 50>$ me promit de venir me rendre sa visite pour $\mid$ en meme temps visiter mes travaux.

» mais chemin faisant dans les champs ${ }^{90} \mid$ je me disait comme les français sont degenerais ${ }^{91}$ une | foi dans les paÿs étrangérs, car mademande netait rien | autre que celle de le prier de faire connaitre au prince | presidant mon arrivee aux portes de france avec $\mid$ mes travaux reunis durent la serie $<$ ? $>$ de 47 ans sur | les trois parties du monde, de la on voit que tres souvent $\mid$ les reputations quelles ne sont que surpées et non | réelles, tel quon veut bien le dire ou se plait adire | tel que j'ais eu au casion de les juger moi même | an visitent personnelement l'objet de la renommee | exsurpée, car le général dufour ma declairait $\mid$ quil se repuinait $<$ ? $>92$ areclamer se qui le consernait $\mid$ et quil prefairait la bandonner antourrent $<$ ? $>\mid$ des chauses de ce monde et que cetet pour ce | la quil vivait loin des hommes et des chauses $\mid$ je vis bien par la que cenetait pas tout se que $\mid$ les génevois me chantait sur le herau de $\mid$ sandeboun $^{93}$.

»et que se général laurait comme les autres afaire | sa cour a son eleve devenus suisses et puisse $<$ ? $>\mid$ devenus français, et placé ala tete de la France $\mid<$ verso :> cest vrais quon voit toujour mieux de pres $\mid$ que tout se qui luit nest pas del'or, tel que sur $\mid$ la feme ou les rubis et les perles ne sont que $\mid$ de verres, mais qui produisent leffet du naturel | par leluision qui procurait de loin, il en est $\mid$ du meme des hommes lors quon les voit depres

\footnotetext{
${ }^{89}$ En juillet 1852, Dufour est âgé de 64 ans.

${ }^{90}$ Le général habitait sa villa de Contamines, située à moins de dix minutes (à pied) du centreville...

${ }^{91}$ Pour « dégénérés »?

92 Pour « répugnait »?

93 Allusion à la guerre du Sonderbund (1847).
} 
» du reste le général dufour fut $\mid$ tres polis et même tres confiant en vers moi | en me citant des faits qui le consernait relative|ment au gouvernemen français et sareserve $\mid$ reserve $<$ sic $>$ en vers le president de cette $\mid$ republique, tout en le quitant lors quil | macompagniet du peron de son jardin \& | lui faisant compliment de son bon chois $\mid$ pour sa residance, il me repondit cest ici $\mid$ que je vis loin des hommes, et des chauses de $\mid$ ce monde, et $\vee$ je lui repondit et vous pouvez $\mid$ medité en paix, le general souffrait dune $\mid$ oppression du cote droit qui le privait par | intervale de respirer avec facilites et lobliget | de garder le repau. Se sont toujour des relicues | qui a compagniant les vieux ages du militaire | et ou on a toujour quelques resantimens. se qui | est d'asses lusage. »

On dit que la civilisation a fait de gros progrès depuis les âges barbares, mais elle a aussi suscité une aggravation de l'égoïsme à un degré sublime. Comme il y a tant de réputation fausse et usurpée, je voulus connaître si celle que les Suisses accordaient au général Dufour était bien méritée en ce qui concerne son nationalisme et sa solidarité pour ses compatriotes. On me les louait, ainsi que son extrême obligeance envers ceux qui avait recours à lui dans certaines circonstances dans son nouveau pays d'adoption, et on me le citait comme un homme bon, obligeant et surtout très pieux, etc.

Après 47 années de voyages en Afrique, Asie et Europe, en juillet 1852, je me trouvais à Genève où se terminait mes 47 années de voyages. Suivant les conseils qu'on me donnait, je voulus aussi rendre visite à ce vétéran de l'armée française, et je me trouvai en conférence avec son excellence, en tête-à-tête dans son cabinet, lui faisant part de mon arrivée et de mes longs voyages. Je le priai qu'il puisse m'obliger à en faire part à son tour au président de la république française, après qu'il aura vu la quantité de travail sur les trois parties du monde où j'avais consacré la plus grande partie de ma vie en y rassemblant tant de matériaux, tant en dessins qu'en manuscrits et que j'étais désireux de rentrer en France.

À cette demande, le général Dufour se retrancha derrière la barrière de sa phraséologie : « J'ai tant pris sur moi envers le président qu'aujourd'hui je m'abstiens autant que possible de lui écrire et de lui demander quoi que ce soir en faveur des sciences, des lettres et des beaux-arts, vu que tout le monde se tournait vers lui pour être son obligé ». Mais que j'étais assez connu des savants et des académies de France pour obtenir ce que je désirais et que je devais leur écrire directement. Là, je vis à qui j'avais affaire. Je répondis comme lui, ou du moins dans le même sens. Et je me disais «C'est assez causé, je vois à qui j'ai affaire ». Même si les Suisses m'avaient dit que le général avait beaucoup appris depuis qu'il étudiait, bien qu'arrivé à l'âge de 70 ans, je ne savais pas encore quelle discipline $<$ ? $>$.

Notre conférence dura trois quarts d'heure, après quoi je pris congé de son excellence suisse et je le quittai au pas de la porte de son jardin, où il me promit de venir me rendre ma visite pour visiter mes travaux en même temps.

Mais, chemin faisant, je me disais que les Français sont dégénérés une fois dans les pays étrangers. Car ma demande n'était rien d'autre que celle de le prier de faire connaître au 
prince-président mon arrivée aux portes de France avec mes travaux réunis durant 47 ans sur les trois parties du monde. De là, on voit que les réputations sont très souvent usurpées et non réelles, comme on veut bien le dire ou qu'on se plaît à dire, et comme j'ai eu l'occasion d'en juger par moi-même, en rendant visite personnellement à une personne dont la renommée est usurpée. Car le général Dufour m'a déclaré qu'il se répugnait à réclamer ce qui le concernait et qu'il préférait l'abandonner en se détournant $<$ ? $>$ des choses de ce monde, et que c'était pour cela qu'il vivait loin des hommes et des choses. Je vis bien par-là que ce n'était pas tout ce que les Genevois me chantaient sur le héros du Sonderbund.

Et que ce général aurait comme les autres à faire sa cour à son élève devenu suisse puis devenu français et placé à la tête de la France. Il est vrai qu'on voit toujours mieux de près que tout ce qui luit n'est pas de l'or. De même que sur les femmes, les rubis et les perles qui ne sont qu'en verre produisent l'effet du naturel par l'illusion qu'ils procurent de loin, il en est de même des hommes lorsqu'on les voit de près.

Du reste, le général Dufour fut très poli et même très confiant envers moi, en me citant des faits qui le concernaient à propos du gouvernement français et à propos du président de cette république. En le quittant, alors qu'il m'accompagnait sur le perron de son jardin et que je lui faisais compliment sur le bon choix de sa résidence, il me répondit : "c'est ici que je vis loin des hommes et des choses de ce monde». Je lui répondis : «Et vous pouvez méditer en paix ». Le général souffrait d'une oppression du côté droit qui le privait par intervalle de respirer avec facilité et l'obligeait à garder le repos. Ce sont toujours des reliques qui accompagnent les militaires âgés, qu'ils ressentent toujours un peu, ce qui est assez habituel. 